\title{
Mathematical modeling of heat transfer in production premises heated by gas infrared emitters
}

\author{
Vyacheslav I. Maksimov ${ }^{1}$, Aleksey M. Bubenchikov ${ }^{2}$, Tatiana A. Nagornova ${ }^{1,}$, and Alime \\ A. Dudak ${ }^{1}$ \\ ${ }^{1}$ National Research Tomsk Polytechnic University, 634050 Tomsk, Russia \\ ${ }^{2}$ Tomsk State University, 634050 Tomsk, Russia
}

\begin{abstract}
The results of numerical modeling of the process of free convective heat transfer in the regime of turbulent convection in a closed rectangular region heated by an infrared radiator are presented. The system of Navier-Stokes equations in the Boussinesq approximation is solved, the energy equation for the gas and the heat conduction equations for the enclosing vertical and horizontal walls. A comparative analysis of the heat transfer regimes in the considered region for different Grashof numbers is carried out. The features of the formation of heated air flows relative to the infrared emitter located at some distance from the upper horizontal boundary of the region are singled out.
\end{abstract}

\section{Introduction}

Despite the indisputable prospect of using gas infrared emitters (GIE) [1] to create a comfortable thermal regime in the local zones of large-sized production facilities, such heating systems are rarely used until now. This is due to the lack of methods for calculating the temperature fields in the areas of operation of the GIE, taking into account the main significant factors. Mathematical models [2] and the corresponding calculation methods are developed for the conditions for placing the radiators under consideration directly on the surface of the upper floors of the rooms, in which the heated GIE local zones are allocated. In real practice, the most efficient radiators can be displaced relative to the upper boundary of the heated area (Figure 1) and as close as possible to the specific workplace (or places).

\footnotetext{
* Corresponding author: tania@tpu.ru
} 


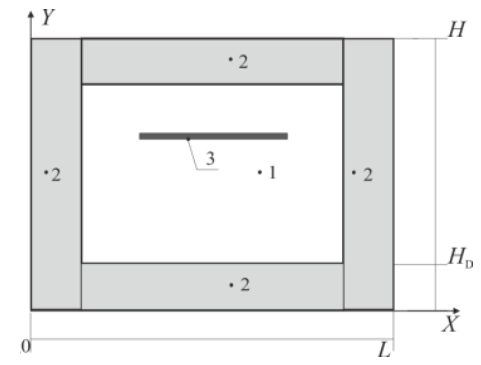

Fig. 1. Area of solution of the problem under consideration: 1 - air; 2 - enclosing structures; 3 infrared emitter; $L, H$ - size of the area in the direction $X$ and $Y$.

When implementing this option, a significant reduction in energy costs for heating one workplace is possible. For these reasons, it is of interest to develop models [2] on the region of more complex free-convective flows corresponding to the geometry shown in Fig. 1.

The purpose of this work is to simulate the mathematical simulation of the conjugate heat transfer in a region limited by vertical and horizontal enclosing structures of finite thickness with a gas infrared radiator that is substantially displaced vertically relative to the upper boundary of the analysis area (Fig. 1).

\section{Statement of the problem and method of solution}

In the formulation of the problem, it was assumed that the air in the region under consideration is transparent to the radiation by the medium, and heat transfer is due to natural convection. It is also assumed that the radiation flux generated by the GIE is completely absorbed at the lower boundary of the region. Heating of the vertical walls is carried out by the flow of streams heated at the lower boundary of the air in the $Y$ direction. The heat sink was taken into the enclosing structures and the accumulation of energy in them. The axisymmetric problem was solved. The gas infrared emitter was a rectangle of finite thickness. Based on the experimental data, boundary conditions of the first kind were established at all boundaries of the GIE. It is established that during operation such radiators are heated up to sufficiently high temperatures (up to $250{ }^{\circ} \mathrm{C}$ ), which remain constant for long periods of time.

The results of solving a group of analogous problems of conjugate heat transfer in closed regions of small dimensions with local energy sources [3-5] have shown that the formation of temperature fields in regions with walls accumulating heat is significant. Therefore, the problem of non-stationary heat transfer was solved. The thermal conductivity and convection in the gas, as well as the thermal conductivity in the enclosing structures were taken into account.

An analysis of the ranges of changes in the main characteristics and parameters of the processes under consideration, as well as the typical sizes of the regions in which GIE is possible, showed that the Gr numbers in real conditions exceed $10^{10}$. That is, the regimes of thermogravitational convection under the conditions under consideration are turbulent. Accordingly, the statement of the problem was carried out taking this factor into account. A $\mathrm{k}-\varepsilon$ model of turbulence was used $[5,6]$.

\section{Mathematical model and method of solution}

In the framework of the physical formulation described above, a mathematical model is formulated that includes Reynolds averaged Navier-Stokes equations, the energy equation 
for the gas, and the heat conduction equations for the enclosing structures with the corresponding boundary conditions below.

$$
\begin{gathered}
\frac{\partial \Omega}{\partial \tau}+U \frac{\partial \Omega}{\partial X}+V \frac{\partial \Omega}{\partial Y}=\frac{\partial^{2}}{\partial X^{2}}\left[\left(\frac{1}{\sqrt{G r}}+\frac{1}{\operatorname{Re}_{t}}\right) \Omega\right]+\frac{\partial^{2}}{\partial Y^{2}}\left[\left(\frac{1}{\sqrt{G r}}+\frac{1}{\operatorname{Re}_{t}}\right) \Omega\right]+\frac{\partial \Theta}{\partial Y}, \\
+2 \frac{\partial U}{\partial Y} \frac{\partial^{2}}{\partial X^{2}}\left(\frac{1}{\operatorname{Re}_{t}}\right)-2 \frac{\partial V}{\partial X} \frac{\partial^{2}}{\partial Y^{2}}\left(\frac{1}{\operatorname{Re}_{t}}\right)+2\left(\frac{\partial V}{\partial Y}-\frac{\partial U}{\partial X}\right) \frac{\partial^{2}}{\partial X \partial Y}\left(\frac{1}{\operatorname{Re}_{t}}\right) \\
\frac{\partial \Theta}{\partial \tau}+U \frac{\partial \Theta}{\partial X}+V \frac{\partial \Theta}{\partial Y}=\frac{\partial}{\partial X}\left[\left(\frac{1}{\operatorname{Pr} \sqrt{G r}}+\frac{1}{\operatorname{Pr}_{t} \operatorname{Re}_{t}}\right) \frac{\partial \Theta}{\partial X}\right]+\frac{\partial}{\partial Y}\left[\left(\frac{1}{\operatorname{Pr} \sqrt{G r}}+\frac{1}{\operatorname{Pr}_{t} \operatorname{Re}_{t}}\right) \frac{\partial \Theta}{\partial Y}\right], \\
\frac{\partial^{2} \Psi}{\partial X^{2}}+\frac{\partial^{2} \Psi}{\partial Y^{2}}=-2 \Omega, \\
\frac{\partial E}{\partial \tau}+U \frac{\partial E}{\partial X}+V \frac{\partial E}{\partial Y}=\frac{\partial}{\partial X}\left[\left(\frac{1}{\sqrt{G r}}+\frac{1}{\sigma_{\varepsilon} \operatorname{Re}_{t}}\right) \frac{\partial E}{\partial X}\right]+\frac{\partial}{\partial Y}\left[\left(\frac{1}{\sqrt{G r}}+\frac{1}{\sigma_{\varepsilon} \operatorname{Re}_{t}}\right) \frac{\partial E}{\partial Y}\right]+ \\
+c_{1 \varepsilon}\left(\tilde{P}_{k}+c_{3 \varepsilon} \tilde{G}_{k}\right) \frac{E}{K}-c_{2 \varepsilon} \frac{E^{2}}{K}, \\
\frac{\partial K}{\partial \tau}+U \frac{\partial K}{\partial X}+V \frac{\partial K}{\partial Y}=\frac{\partial}{\partial X}\left[\left(\frac{1}{\sqrt{G r}}+\frac{1}{\sigma_{k} \operatorname{Re}_{t}}\right) \frac{\partial K}{\partial X}\right]+\frac{\partial}{\partial Y}\left[\left(\frac{1}{\sqrt{G r}}+\frac{1}{\sigma_{k} \operatorname{Re}_{t}}\right) \frac{\partial K}{\partial Y}\right]+ \\
+\tilde{P}_{k}+\tilde{G}_{k}-E \\
\frac{1}{\mathrm{Fo}_{0} \frac{\partial \Theta}{\partial \tau}}=\frac{\partial^{2} \Theta}{\partial X^{2}}+\frac{\partial^{2} \Theta}{\partial Y^{2}}, .
\end{gathered}
$$

where $\tilde{P}_{k}=\frac{1}{\operatorname{Re}_{t}}\left[2\left(\frac{\partial U}{\partial X}\right)^{2}+2\left(\frac{\partial V}{\partial X}\right)^{2}+\left(\frac{\partial U}{\partial X}+\frac{\partial V}{\partial X}\right)^{2}\right] ; \quad \tilde{G}_{k}=-\frac{1}{\operatorname{Re}_{t} \operatorname{Pr}_{t}} \frac{\partial \Theta}{\partial Y}$; $=1$.

The model constants are given as: $\mathrm{c}_{\mu}=0,09 ; \mathrm{c}_{1 \varepsilon}=1,44 ; \mathrm{c}_{2 \varepsilon}=1,92 ; \mathrm{c}_{3 \varepsilon}=0,8 ; \sigma_{\varepsilon}=1,3 ; \sigma_{\mathrm{\kappa}}$

Initial conditions for the system of equations (1-4):

$$
\Psi(X, Y, 0)=\Omega(X, Y, 0)=\Theta(X, Y, 0)=K(X, Y, 0)=E(X, Y, 0)=0
$$

Boundary conditions at the three external borders of region solutions:

$$
\frac{\partial \Theta(X, Y, \tau)}{\partial n}=0 .
$$

At the interfaces of solid walls and gas the following conditions are fulfilled:

$$
\frac{\partial \Psi(X, Y, \tau)}{\partial n}=0, \frac{\partial \Theta_{1}(X, Y, \tau)}{\partial n}=\lambda_{1,2} \frac{\partial \Theta_{2}(X, Y, \tau)}{\partial n}, \Theta_{1}(X, Y, \tau)=\Theta_{2}(X, Y, \tau) .
$$

On the surface of the radiator:

$$
\Theta_{1}(X, Y, \tau)=1,
$$

At the boundary $H_{\mathrm{D}}$ of the radiation heat source is taken into account:

$$
\frac{\partial \Psi(X, Y, \tau)}{\partial Y}=0, \frac{\partial \Theta_{1}(X, Y, \tau)}{\partial Y}=\frac{\partial \Theta_{2}(X, Y, \tau)}{\partial Y}+\mathrm{Ki}, \Theta_{1}(X, Y, \tau)=\Theta_{2}(X, Y, \tau)
$$

For the $\kappa-\varepsilon$ model near the solid surface the following is accepted: 


$$
\frac{\partial K}{\partial n}=0 ; E=\frac{c_{\mu}^{3 / 4} K^{3 / 2}}{k \Delta n}
$$

where $X, Y$-dimensionless Cartesian coordinates; $\tau$-dimensionless time; $t$ - time, s; $t_{0}-$ time scale, s; $U, V$-dimensionless velocity; $V_{0}$ - velocity scale (convection velocity), $\mathrm{m}^{2} / \mathrm{s}$; $\Theta$ - dimensionless temperature; $T$ - temperature, $\mathrm{K} ; T_{0}$ - temperature at the initial time, $\mathrm{K}$; $T_{i t}$ - heat source temperature, $\mathrm{K} ; \mathrm{g}$ - acceleration created by the mass forces, $\mathrm{m} / \mathrm{s}^{2} ; \beta-$ thermal coefficient of volume expansion, $\mathrm{K}^{-1} ; \psi-$ stream function, $\mathrm{m}^{2} / \mathrm{s} ; \psi_{0}$ - current function scale, $\mathrm{m}^{2} / \mathrm{s} ; \Psi$ - dimensionless analog stream function $\psi ; \omega-$ swirl of vorticity, $1 / \mathrm{c} ; \omega_{0}$ - vorticity scale, $1 / \mathrm{s} ; \Omega$ - dimensionless analog $\omega ; K$ - dimensionless analog of turbulent kinetic energy; $E$ - dimensionless analog of the dissipation rate of turbulent kinetic energy; $\mathrm{Ki}=q L^{3} / \lambda\left(T_{i t}-T_{0}\right)-$ Kirpichev number; $\mathrm{Gr}=g \beta L^{3}\left(T_{i t}-T_{0}\right) / v^{2}-$ Grashof number; $\mathrm{Re}_{t}=E / c_{\mu} K^{2}$ - turbulent Reynolds number; $v_{t}$ - coefficient of turbulent kinematic viscosity, $\mathrm{m}^{2} / \mathrm{s} ; \quad \lambda_{1,2}=\lambda_{2} / \lambda_{1}-$ relative thermal conductivity coefficient; $\operatorname{Pr}=v_{t} / a-$ turbulent Prandtl number; $\operatorname{Pr}=v / a-$ Prandtl number; Fo $=a t_{0} / L^{2}-$ число Фурье; $a-$ thermal diffusivity, $\mathrm{m}^{2} / \mathrm{s}$.

Equations (1-6) with the corresponding initial and boundary conditions (7-11) are solved by the method of finite differences $[7,8]$ on a uniform grid. To solve the system of difference analogs of the initial nonlinear partial differential equations with nonlinear boundary conditions, the method and algorithm developed [3-5] for solving problems of thermogravitational convection in closed regions with local energy sources were used. To substantiate the reliability of the results of numerical modeling, the conservativeness of the difference scheme was estimated by a technique developed for solving nonlinear heat conduction problems [9] and developed for ignition of water-coal fuels under conditions of intense local injection of fumes into the region filled with an oxidizer [10,11].

\section{Analysis of numerical simulation results}

Numerical studies were carried out for free-convective flows corresponding to the turbulent regime for two Grashof numbers: $\mathrm{Gr}=4 \cdot 10^{12}$ and $\mathrm{Gr}=10^{10}$. The results obtained with these values of Gr sufficiently clearly demonstrate the main regularities of the processes under consideration. Other used dimensionless and dimensional values $\left(L=4 \mathrm{~m}, t_{0}=5 \mathrm{~s}, T_{0}=293\right.$ $\mathrm{K}, T_{i t}=353 \mathrm{~K}$ ), were also chosen to correspond to the actual values of the main characteristics when infrared emitters operate. Numerical studies were carried out at the values of the heat flux arriving at the $H_{D}$ surface (Table 1).

Table. 1. The values of the heat flux over the surface of the concrete base in the section $Y=H_{D}$ for $L / 2<X<0,875 L$ (fig. 1).

\begin{tabular}{|l|l|l|l|l|l|l|l|}
\hline Section & $X=0.5 \mathrm{~L}$ & $X=0.563 \mathrm{~L}$ & $X=0.685 \mathrm{~L}$ & $X=0.688 \mathrm{~L}$ & $X=0.75 \mathrm{~L}$ & $X=0.813 \mathrm{~L}$ & $X=0.875 \mathrm{~L}$ \\
\hline$q, \mathrm{~W} / \mathrm{m}^{2}$ & 190.5 & 180.2 & 182.1 & 172.2 & 134.2 & 122.2 & 102.1 \\
\hline
\end{tabular}

The analysis of thermal regimes in the quite typical region under consideration can be carried out both with respect to the distributed characteristics of the process (inhomogeneous in real practice and nonstationary temperature fields) and by the averaged ones (the temperature averages over the region at fixed instants of time). In the first case, the temperature gradients can be estimated and the maximum or minimum air temperature zones identified. In the second case (which is most often used in practice), it is possible to easily and quickly compare, by average temperature values, several heating regimes, 
differing by the intensity of radiation coming from the GIE or by the position of the radiators relative to the upper ceiling of the room.

Below are the results of numerical modeling of temperature fields in the analysis area in the form of distributions $\Theta$ and mean temperature values.

In Fig. 2 and 3 show typical results of numerical simulation illustrating the main regularities of heat transfer in the heating system under consideration using gas infrared emitters.

Comparing the results of numerical studies for various Grashof numbers, it can be noted that at $\mathrm{Gr}=10^{12}$, a much more intensive circulation of air currents occurs (Fig. 2). In turn, this leads to an increase in heat transfer in the considered region, as evidenced by the mean temperatures (Table 2).

An analysis of the results of numerical modeling of the processes under consideration gives grounds also for conclusions about the possible optimization of heating schemes for workplaces using infrared emitters. So, for example, it is obvious that there is a possibility of significant energy savings in the heat insulation of the air zone between the upper surface of the GIE (Figure 1) and the overlap. It is clearly seen that as a result of thermogravitational convection, the air masses heated up by the heat sink from the lower enclosing structure rise upward, flow around the GIE, move parallel to the upper boundary of the region, giving energy to the overlaps (then an intense heat dissipation into the atmosphere is possible), and descend along the vertical enclosing structures. Obviously, a significant part of the energy is absorbed by the slabs because the air reaches them with the highest possible temperature. In addition to the energy transmitted from the heated GIE of the lower ceiling of the room, the air masses when heated up are additionally heated when flowing around the radiator housing.

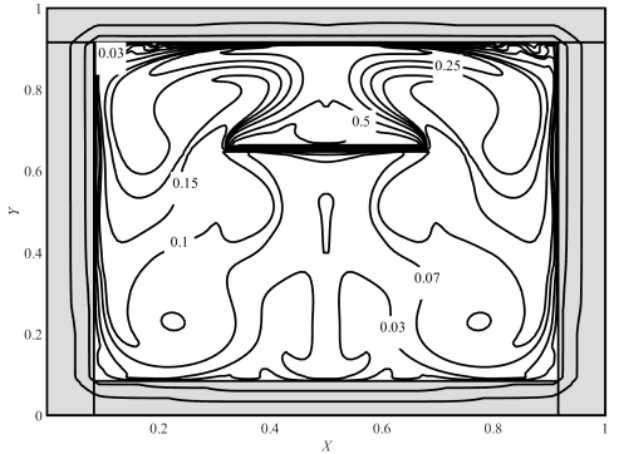

$a$

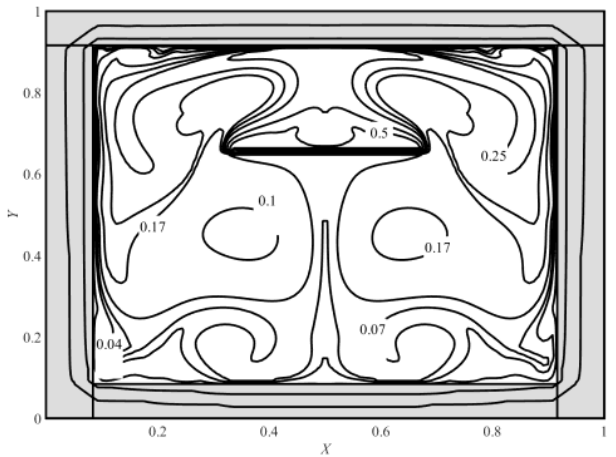

$c$

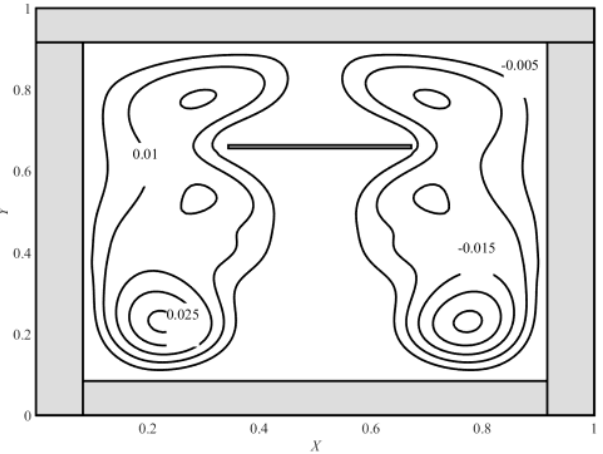

$b$

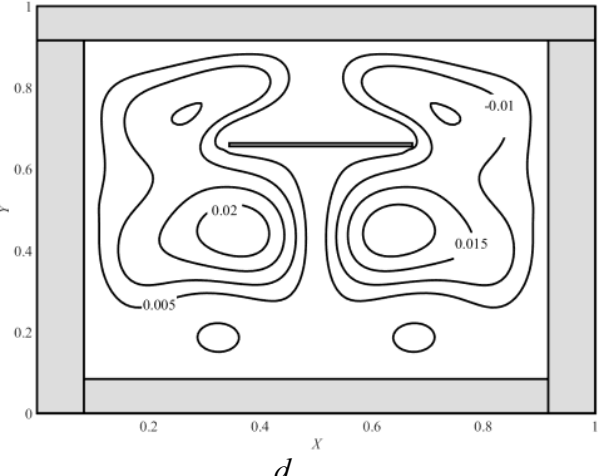

$d$

Fig. 2. Fields of temperatures $(a, c)$ and isolines of the current function $(b, d)$ for natural convection conditions in a closed region for $\mathrm{Gr}=4 \cdot 10^{12}$ for different values of $\tau: \tau=200(a, b), \tau=300(c, d)$. 
Table. 2. Mean temperature values $\Theta$ for different time instants.

\begin{tabular}{|c|c|c|}
\hline$\tau$ & $\mathrm{Gr}=10^{12}$ & $\mathrm{Gr}=4 \cdot 10^{10}$ \\
\hline 200 & 0.147 & 0.148 \\
\hline 300 & 0.171 & 0.188 \\
\hline
\end{tabular}

Isotherms (Fig. 2, 3) illustrate well that the region of the highest temperatures in a room heated by GIE is formed far beyond the working area (located near the lower overlap). In essence, to a large extent, GIE works on heating the air in the upper part of the room and the plates of the upper ceiling. At the same time, the losses of thermal energy are great.

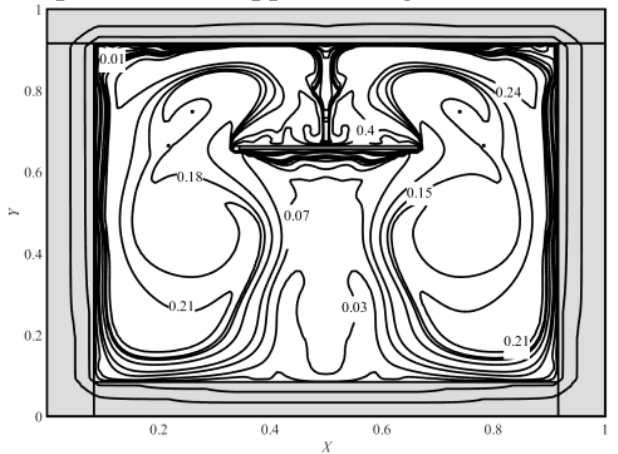

$a$

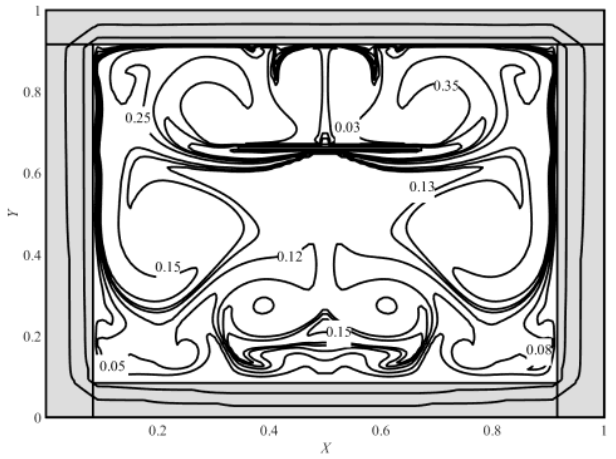

C

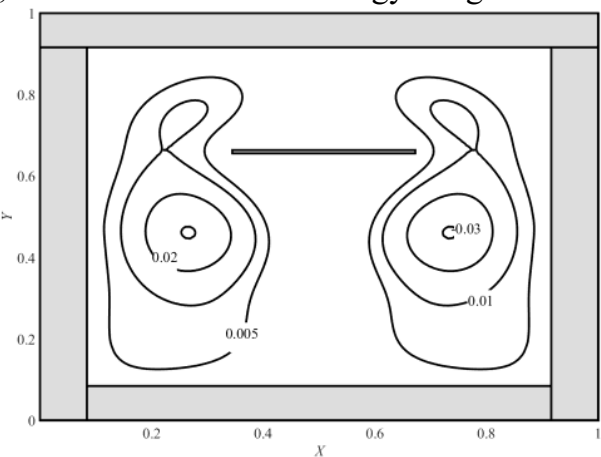

$b$

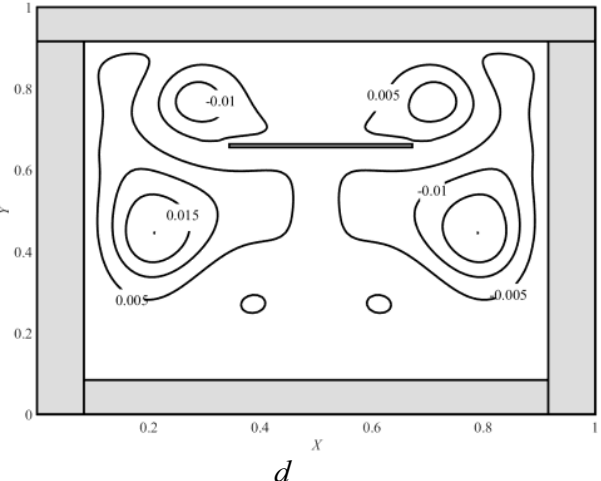

Fig. 3. Fields of temperatures $(a, c)$ and isolines of the current function $(b, d)$ for natural convection conditions in a closed region for $\mathrm{Gr}=4 \cdot 10^{10}$ for different values of $\tau: \tau=200(a, b), \tau=300(c, d)$.

It seems reasonable to at least maximally effective thermal insulation of the upper surface of the GIE corps. A significantly greater energy saving effect can be achieved by eliminating the circulating region of hot air above the GIE casing or (at least) the thermal insulation of the plate on their lower surface. Eliminate the circulation of heated air in the upper part of the room by placing obstacles in the path of the air masses moving upwards parallel to the body of the GIE.

\section{Conclusion}

The results obtained during the solution of the problem of thermogravitational convection of air under conditions of intensive energy supply due to the operation of gas infrared radiators and heat removal into the enclosing structures are the basis for the development of methods for calculating the thermal regimes of local working zones in large-sized 
production rooms with heating systems based on GIE. The possible high efficiency of using such radiators for local heating of single and group work stations is shown.

The regularities of heat transfer established under the results of numerical simulation in the conditions of infrared emitters are also a basis for the development of models of the conjugate heat transfer in the part of the transition from traditional productions of computational fluid mechanics problems [3-5] to the tasks of industrial heat and power engineering and energy saving.

The work was supported by the Russian President's grant (Scientific School project 7538.2016.8).

\section{References}

1. K.-N. Rhee, B.W. Olesen, K.W. Kim, Building and Environment, 112, 367 (2017)

2. G.V. Kuznetsov, N.I. Kurilenko, V.I. Maksimov, G.Ya. Mamontov, T.A. Nagornova, J. Eng. Phys. Thermophys. 86 (3), 519 (2013)

3. G.V. Kuznetsov, M.A. Sheremet, Comput. Therm. Sci. 1 (3), 341 (2009)

4. V.I. Maksimov, T.A. Nagornova, I.A. Shestakov, EPJ Web Conf. 82, 01048 (2015)

5. G.V. Kuznetsov, M.A. Sheremet, Int. J. Heat Mass Transfer, 53, 163(2010)

6. V.I. Maksimov, T.A. Nagornova, V.P. Glazyrin, EPJ Web Conf. 110, 01038 (2016)

7. D.S-K. Ting, Basics of engineering turbulence (Academic Press, USA, 2016)

8. T. Cebeci, Analysis of Turbulent Flows with Computer Programs (Netherlands, Elsevier Ltd., 2013)

9. V.L. Strakhov, A.N. Garashchenko, G.V. Kuznetsov, V.P. Rudzinskij, Combustion, Explosion, and Shock Waves, 37 (2), 178 (2001)

10. G.V. Kuznetsov, V.V. Salomatov, S.V. Syrodoy, Combustion, Explosion and Shock Waves, 51 (4), 409 (2015)

11. G.V. Kuznetsov, V.V. Salomatov, S.V. Syrodoy, Thermal Engineering, 62 (10), 703 (2015) 